\title{
Mécanismes de Biosynthèse et de Régulation des Glucosinolates
}

\author{
Malika Ouassou, \\ Université Abdel Malek Essaidi, Faculté des Sciences et Techniques de \\ Tanger, Equipe de Biochimie et Génétique Moléculaire Tanger, \\ Maroc Université Paris Saclay, Laboratoire Ecologie Systèmatique et \\ Evolution, Orsay, France \\ Amal El Amrani, \\ Université Abdel Malek Essaidi, \\ Faculté des Sciences et Techniques de Tanger \\ Equipe de Biochimie et Génétique Moléculaire.Tanger, Maroc
}

\section{Maisara Mukhaimar,}

Centre National Palestinien de la recherche Agronomique (NARC),

Ministère de l'Agriculture, Gaza, Palestine

Doi: 10.19044/esj.2019.v15n3p313～URL:http://dx.doi.org/10.19044/esj.2019.v15n3p313

\section{Résumé}

Les glucosinolates des produits naturels riches en soufre qui, après hydrolyse par des thioglucosidases appelées myrosinases, produisent plusieurs produits différents (isothiocyanates, thiocyanates et nitriles), ces produits d'hydrolyse ont de nombreuses propriétés biologiques différentes, notament dans la défense des plantes contre les attaques des ennemis naturels. Nous résumons ici des études menées chez Brassica qui ont fourni des preuves moléculaires sur la biosynthèse des glucosinolates leurs régulations à différent niveaux et le contrôle génétique de la variation naturelle de leur accumulation.

Mots clés: Glucosinolates, myrosinases, biosynthèse, variation naturelle, régulation 


\title{
Mechanisms of Biosynthesis and Regulation of Glucosinolates
}

\author{
Malika Ouassou, \\ Université Abdel Malek Essaidi, Faculté des Sciences et Techniques de \\ Tanger, Equipe de Biochimie et Génétique Moléculaire Tanger, \\ Maroc Université Paris Saclay, Laboratoire Ecologie Systèmatique et \\ Evolution, Orsay, France \\ Amal El Amrani, \\ Université Abdel Malek Essaidi, \\ Faculté des Sciences et Techniques de Tanger \\ Equipe de Biochimie et Génétique Moléculaire.Tanger, Maroc
}

\section{Maisara Mukhaimar,}

Centre National Palestinien de la recherche Agronomique (NARC),

Ministère de l'Agriculture, Gaza, Palestine

\begin{abstract}
Glucosinolates are natural products sulfur-rich that upon hydrolysis by thioglucosidases called myrosinases produce several different products (isothiocyanates, thiocyanates, and nitriles), these hydrolysis products have many different biological properties, especially in the defense of plants against the attacks of natural enemies. Here we summarize studies in Brassica that have provided molecular evidence on the biosynthesis of glucosinolates, their regulation at different levels and the genetic control of the natural variation of their accumulation.
\end{abstract}

Keywords: Glucosinolates, myrosinases, biosynthesis, natural variation, regulation

\section{Introduction}

Dans la nature, les plantes sont constamment confrontées à des contraintes environnementales, telles que la sécheresse, les variations de températures, les blessures physiques, les attaques des agents pathogènes d'origine diverse (bacterienne, virale, fongique...) et les attaques des insectes ravageurs (Howe et Jander, 2008). Pour faire face au stress environnemental, les plantes synthétisent un grand nombre de substances chimiques, appelées métabolites secondaires (Croteau et al., 2000). Elles dérivent de métabolites primaires qui ont une fonction vitale pour les 
organismes vivants (protéines, lipides, glucides) (Iriti et Faoro, 2009). A ce jour, plusieurs dizaines de milliers de métabolites secondaires ont été déjà isolés et identifiés. Il y’a quelque décennie, ils étaient considérés comme des molécules sans fonction, mais les études emmenées sur ces métabolites ont prouvé que beaucoup de ces molécules possèdent des fonctions bien précises dans les relations entre les plantes et leur environnement (Richter, 1993). En effet, ils protègent les plantes contre les attaques des herbivores et d'agents pathogènes, ils servent comme phytohormones ou encore comme substances signales pour pollinisateurs et disséminateurs (Kimura et al., 2001; Hartmann, 2007). A ces fonctions, s'ajoute leur utilisation par l'homme comme additifs alimentaires, dans l'industrie pharmaceutique ou encore en agrochimie (Newman et Cragg, 2012). La fonction d'une grande majorité de métabolites secondaires reste néanmoins inconnue.

Les métabolites secondaires peuvent être classés en trois grandes classes: les alcalö̈des, les terpènes et les composés phénoliques, d'autres classes de métabolites secondaires comprennent: les glycosides, les composés azotés et les composés soufrés comme les glucosinolates qui font l'objet de cette étude. Les glucosinolates sont donc des composés riches en soufre, présents dans de nombreux végétaux, essentiellement chez les plantes appartenant à l'ordre des Brassicales, et plus particulièrement sont caractéristiques de la famille des Brassicaceae (Halkier et Gershenzon 2006).

\section{Glucosinolates}

Par leur importante présence dans de nombreux légumes, les glucosinolates font partis de la vie humaine depuis des milliers d'années (Halkier et Gershenzon, 2006). Ils sont à l'origine de la saveur piquante des graines de moutarde. Les premières observations sur les glucosinolates et leurs produits d'hydrolyse, communément appelés les huiles de moutarde, ont été enregistrés au début du $17^{\text {ème }}$ siècle. La sinigrine (2-propenyl ou allyl glucosinolate) et la sinalbine (4-hydroxybenzyl glucosinolate) ont été isolés à partir des graines de la moutarde noire (Brassica nigra) et blanche (Sinapis alba) respectivement dans les années 1830 (Robiquet et Boutron, 1831).

La structure de glucosinolate (Figure 1) consiste en un assemblage d'un thiohydroximate- $O$-sulfonate, d'un glucose et d'une partie relativement hydrophobe $(\mathrm{R})$ qui constitue le seul variant structural (Agerbirk et Olsen, 2012). 


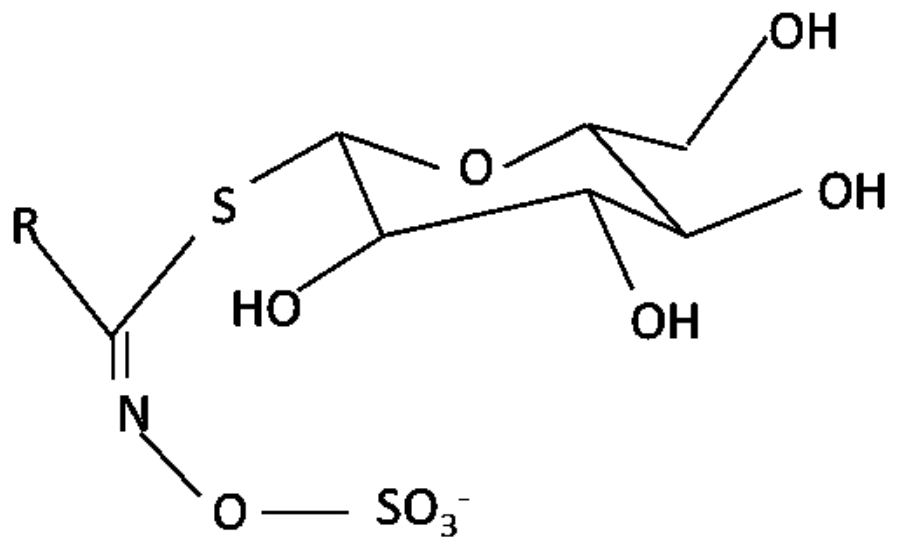

Figure 1. Structure générale de glucosinolate.

Chaine latérale, $R$, est dérivée à partir d'acides aminés.

Les glucosinolates regroupent plus de 130 molécules issues du métabolisme secondaire des plantes de l'ordre des Brassicales (Chen et Andreasson, 2001). Parmi les familles appartenant à cet ordre, celle des Brassicaceae présente un intérêt agronomique et économique important, car elle regroupe de nombreuses espèces considérées comme denrées alimentaires pour l'Homme ou le bétail dont Brassica oleracea (choux divers dont chou-fleur et brocoli), Raphanus sativum (radis), Brassica rapa (navet) ou Brassica napus (colza). Les glucosinolates se trouvent également chez les plantes des familles des Cleomaceae, des Capparaceae et des Resedaceae (Rodman, 1980 ; Rodman et al., 1981 ; Daxenbichler et al., 1991). Ils ont été détectés aussi dans des genres complètement indépendants, tels que dans un petit nombre de plantes du genre Drypetes de la famille des Euphorbiaceae, mais l'origine des glucosinolates est indépendante de celle des Brassicales (Rodman, 1981 ; Halkier et Gershenzon, 2006). Les glucosinolates sont connus par leur rôle de réserve en azote et en soufre pour la plante, notamment pour la synthèse d'acides aminés en cas de nutrition carencée (Schnug, 1990). Des études menées sur des puces à ADN ont montré que l'activation de l'acquisition de soufre et la répression de la biosynthèse des glucosinolates peut se produire parallèlement en réponse à une carence en soufre (Hirai et al., 2005). En outre, certains glucosinolates pourraient servir de réserve pour la production d'acide indolacétique (IAA) et joueraient donc un rôle dans le métabolisme hormonal de la plante (Rausch et al., 1983). On peut aussi rencontrer une diversité importante à l'intérieur d'une même espèce et aussi entre espèces différentes comme cela a été montré par Sang et Truscott (1984) ayant souligné une variation de la concentration en glucosinolates entre les graines, les feuilles et les racines de différentes plantes (Mikkelsen et al., 2002 ; Petersen et al., 2002 ; Brown et al., 2003). Ces métabolites secondaires diffèrent également par leur teneur au cours du cycle 
végétatif (Grubb et Abel, 2006). En effet, il a été montré que la teneur en glucosinolates chez le colza s'avère maximale lors des périodes de croissance et diminue par la suite, par exemple de la gluconapine (but-3-ényle glucosinolate) qui augmente régulièrement, alors que celle de la 4hydroxyglucobrassicine diminue dans les organes végétatifs et augmente dans les organes reproducteurs; la teneur de la progoitrine (3-butenyl glucosinolate) elle augmente quant à elle dans tous les organes (Iori et al., 1990).

Les niveaux de glucosinolates varient à différents stades de développement de la plante et sont affectés par plusieurs facteurs biotiques et abiotiques tels que les conditions de croissance, les infections fongiques ou bactériennes, les blessures causés par les insectes ou par d'autres formes de stress biotiques. D'autres facteurs indirects tels que le stress hydrique (sécheresse ou inondations) et thermique peuvent avoir également une influence sur la teneur en glucosinolates dans la plante (Hasegawa et al., 2000). En général, les niveaux élevés de glucosinolates se trouvent dans les jeunes feuilles, les pousses, les siliques et dans les graines mûres (Bennett et al., 1995; Brown et al., 2003). Les glucosinolates sont normalement hydrophiles, stables et stockés dans des vacuoles de la plupart des tissus végétaux.

Les glucosinolates possèdent aussi un rôle important au point de vue médical. En effet, des études d'épidémiologie suggère que la consommation des plantes de la famille des Brassicaceae réduit le risque d'atteinte du cancer des poumons et du tube digestif (Block et al., 1992 ; Verhoeven et al., 1997 ; Van Poppel et al., 1999). Cependant, seulement quelques glucosinolates avec des propriétés anticancéreuses ont été étudiées, en particulier chez le brocoli (London et al., 2000). Des études menées sur cette espèce en comparant des régimes alimentaires différents ont démontré que le 3méthylsulfinylpropyl (3-MSP) et le 4-méthylsulfinylbutyl (4-MSB) isothiocyanates dérivés de glucosinolates correspondants ont un effet anticancereux et un effet sur des maladies chroniques en modulant le statut redox cellulaire et la fonction de certaines protéines (Mithen et al., 2003). De plus, les produits de dégradation de glucosinolates ont un rôle dans la simulation de l'arrêt de cycle cellulaire et ensuite l'apoptose des cellules cancéreuses. Les glucosinolates jouent un rôle important dans les interactions plantesparasites. Il existe une bonne évidence que ces composés secondaires sont impliqués dans la défense directe ou indirecte des plantes contre certains parasites, mais les glucosinolates ou leur produits d'hydrolyses peuvent aussi stimuler le comportement nutritif et ovipositionnel des insectes et leur oviposition (Mewis et al., 2002 ; Miles et al., 2005 ; De Vos el al., 2008 ; Sun et al., 2009).

La fonction biologique des glucosinolates est donc de défendre les plantes contre les herbivores et les agents pathogènes à travers l'action de 
leurs produits de dégradation. Ils sont présents dans toutes les parties de la plante (Fenwick et al., 1983), spécialement dans des vacuoles et libérés lors d'une attaque d'un herbivore, d'un agent pathogène ou d'une blessure. Quand les tissus sont endommagés, les glucosinolates entrent en contact avec des thioglucosidases spécifiques, appelées myrosinases qui catalysent l'hydrolyse des glucosinolates. La stabilité des glucosinolates dans les tissus intacts est due au fait de leur séparation spatiale avec les myrosinases. Les myrosinases sont des enzymes de 125 à 150 kDa (Bones et Slupphaug, 1989). Elles sont présentes dans tous les tissus des plantes Crucifères, leurs niveau d'activité décroit tout au long de développement de la plante (Iversen et Baggerud, 1980). Elles sont localisées dans des idioblastes, appelées cellules myrosines, qui sont réparties dans les feuilles, racines et tiges. Chez Arabidopsis thaliana, quatre gènes codants pour des myrosinases classiques ont été détectées, TGG1 (At5g26000), TGG2 (At5g25980), TGG4 (At1g47600) et TGG5 (At1g51470) (Xu et al., 2004), deux autres gènes pourraient être codés pour des myrosinases TGG3 (At5g48375) et TGG6 (At1g51490), mais ils sont désignés comme des pseudogènes (Wang et al., 2009). L'analyse métabolique du double mutant knockout $\operatorname{tgg} 1$ / tgg2 a révélé la perte d'activité de la myrosinase liée à l'absence de dégradation des glucosinolates, principalement de glucosinolates aliphatiques. L'acide ascorbique est capable de moduler l'activité des myrosinases en exerçant un effet activateur sur son site actif (Bones et Slupphaug, 1989). En effet, une concentration de $0,1 \mathrm{mM}$ et $10 \mathrm{mM}$ d'acide ascorbique réduit l'activité de la myrosinase. L'excès de l'acide ascorbique agit par effet compétitif à l'égard des glucosinolates pour le centre actif de la myrosinase. La température élevée peut également affecter l'activité des myrosinases comme cela été montré chez la moutarde. D'autres myrosinases dites atypiques ont été découvertes chez A. thaliana telles que PEN2 pour Penetration 2 qui ont une fonction antifongique et PYK10 dont les profils d'expression montrent leur implication dans la réponse aux stress biotiques et abiotiques (Bednarek et al., 2009 ; Clay et al., 2009).

\section{Système glucosinolate-myrosinase}

A l'attaque d'un herbivore ou d'un agent pathogène, les glucosinolates entrent en contact avec les myrosinases et sont hydrolysés en composés bioactifs à large spectre d'activité antifongique, antibacterienne et insecticide (De Vos et al., 2008). Cette relation enzyme-substrat est la clé des dispositifs de défense de plantes de l'ordre des Brassicales, y compris la plante modèle $A$. thaliana contre diverses agressions (Tierens et al., 2001 ; Ratzka et al., 2002 ; Kroymann et al., 2003 ; Kliebenstein et al., 2005). Ce système est un complexe "glucosinolates-myrosinases" (Figure 2), appelé aussi "bombe de l'huile de moutarde". Le mécanisme du système 
glucosinolates-myrosinases permet l'hydrolyse de la liaison thioglucosidique des glucosinolates pour libérer le D-Glucose et le thiohydroximate- $O$-sulfonate (Figure 2), qui libère ensuite le sulfate et se transforme en produits de dégradations (isothiocyanates, thiocyanates, nitriles, epithionitriles et oxazolidine-2-thiones). Certaines conditions chimiques, des co-facteurs ( $\mathrm{pH}$, la disponibilité d'ions ferreux) et la présence de protéines supplémentaires déterminent la structure finale et la composition des produits de dégradation (Lambrix et al., 2001 ; Zabala et al., 2005). Les produits de dégradation des glucosinolates possèdent des activités biocides en raison de leur toxicité pour une variété de pathogènes et herbivores (Fahey et al., 2001 ; Brader et al., 2006).

Attaque des insectes et agents pathogènes
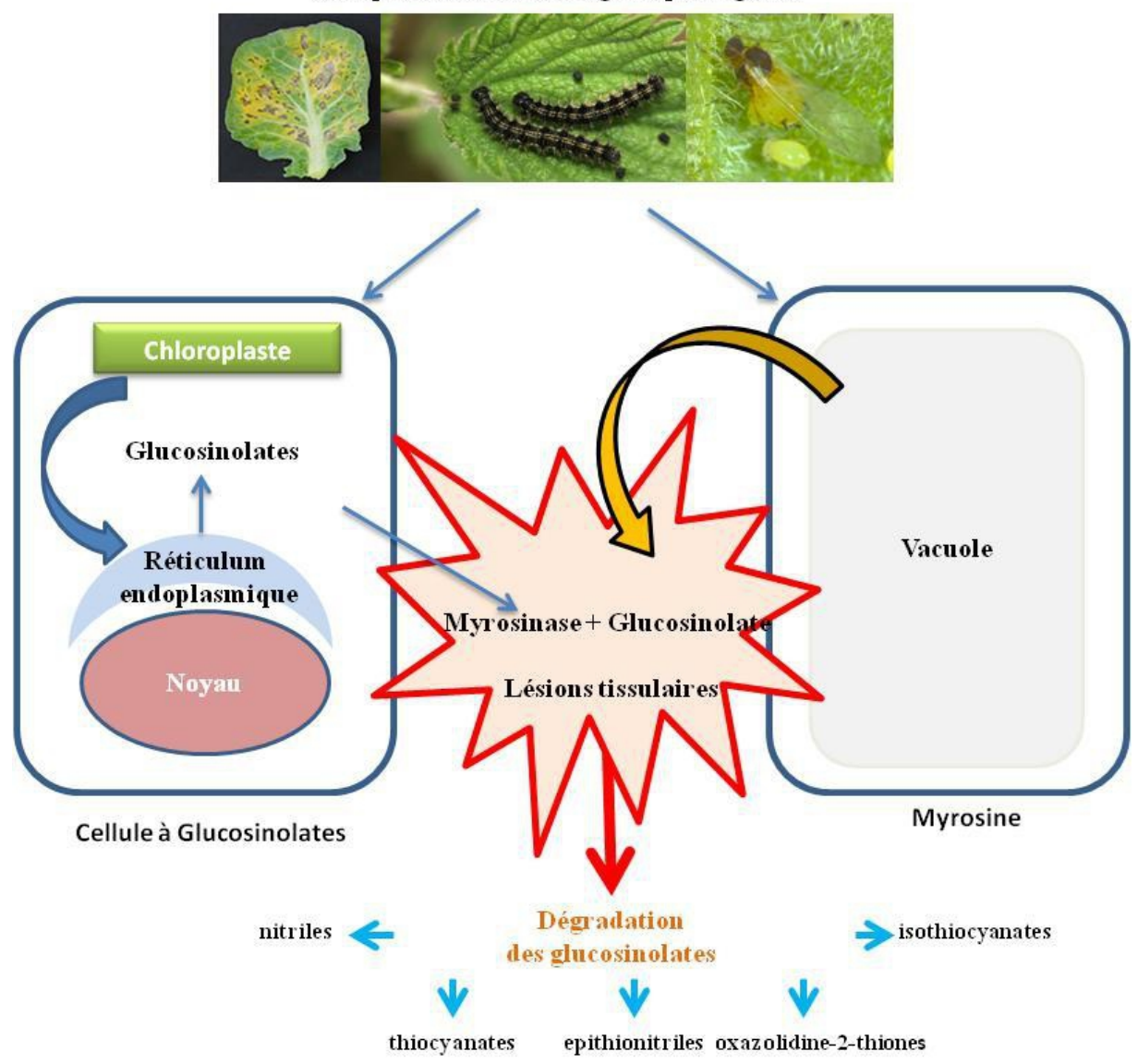

Figure 2. Schéma classique du système glucosinolate-myrosinase.

Les plantes utilisent le système glucosinolates-myrosinase comme système de défense contre des insectes herbivores et des agents pathogènes. Dans les tissus intactes, les glucosinolates et les myrosinases sont séparés spatialement, lors d'une agression de tissu végétal, les glucosinolates stockés 
dans des vacuoles sont libérées et entrent en contact avec les myrosinases en présence de l'eau. Les glucosinolates sont hydrolysés en produits toxiques pour les agresseurs tels que les isothiocyanates, les nitriles, les epithionitriles, les thiocyanates et les oxazolidine-2-thiones (Ratzka et al., 2002).

\section{Biosynthèse des glucosinolates}

Selon l'acide aminé du départ, les glucosinolates peuvent être classés en trois catégories, aliphatiques, aromatiques et indoliques. Les glucosinolates aliphatiques dérivent de la méthionine, alanine, valine, leucine et l'isoleucine, tandis que les glucosinolates aromatiques proviennent de la phénylalanine et de la tyrosine et les glucosinolates indoliques du tryptophane (Halkier et Du, 1997 ; Wittstock et Halkier, 2002 ; Redovnikovic et al., 2008). Chez A. thaliana, la majorité des glucosinolates est synthétisée à partir de la méthionine, phénylalanine et tryptophane (Bennett et al., 1995 ; Kliebenstein et al., 2001a ; Reintanz et al., 2001).

Au cours des dernières années, la biosynthèse des glucosinolates a été largement étudiée chez A. thaliana (Halkier et Du, 1997 ; Mikkelsen et al., 2002 ; Halkier et Gershenzon, 2006 ; Redovnikovic et al., 2008). A partir de la méthionine, elle se décompose en trois grandes étapes (Figure 3), à savoir l'élongation de la chaîne latérale de l'acide aminé méthionine, la formation de la structure de base de glucosinolate et une multitude des modifications secondaires de la chaîne latérale, conduisant à une grande diversité de glucosinolates. A partir de tryptophane et de la phénylalanine, seulement les deux dernières étapes sont réalisées. 


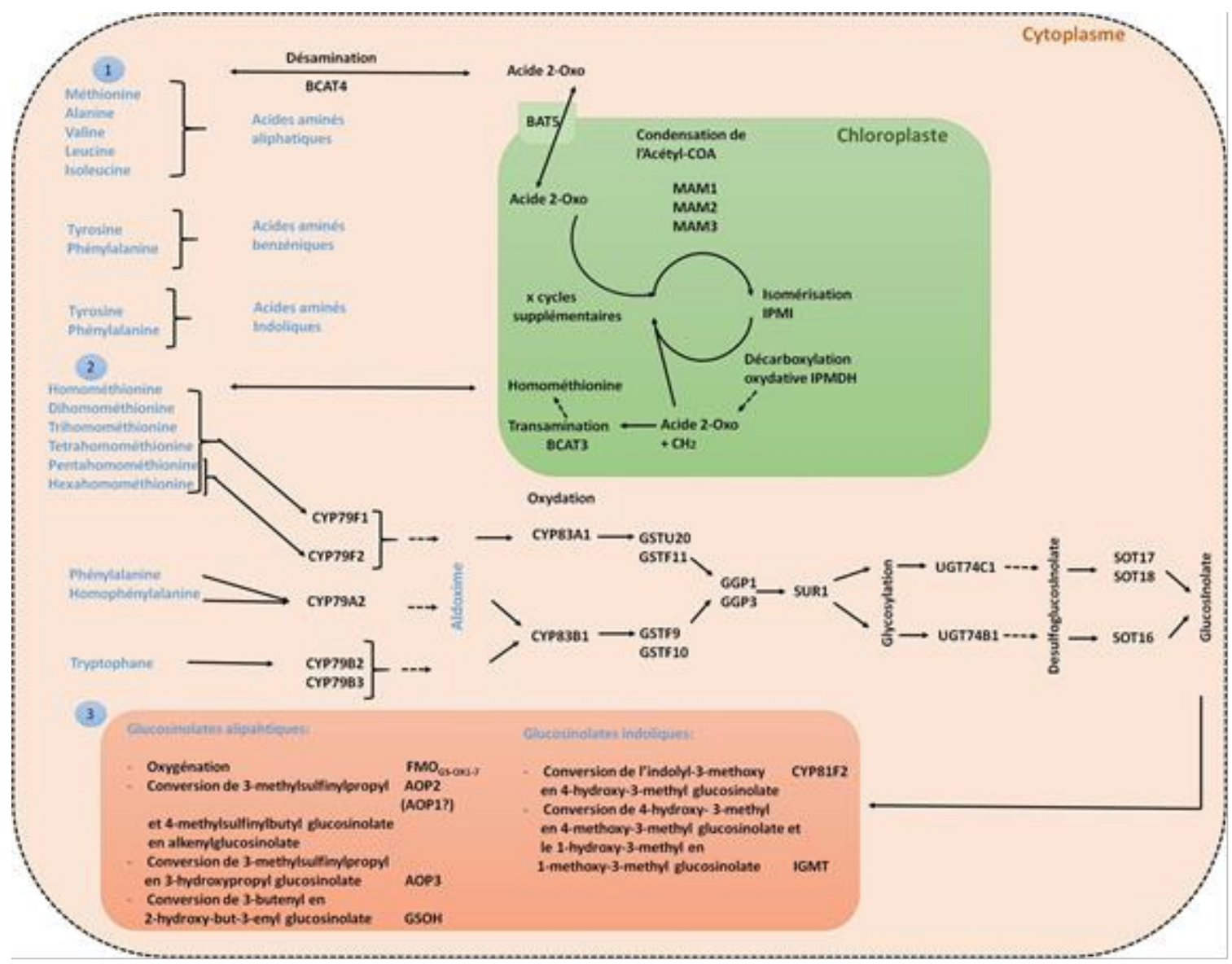

Figure 3. Représentation schématique de la biosynthèse des glucosinolates classée en trois étapes principales.

L'élongation de la chaine latérale de la méthionine (1), la formation de la structure glucosinolate (2) et la modification de la chaine latérale (3). Les enzymes impliqués dans chaque étape sont plus détaillés dans le texte (Sonderby et al., 2010).

\subsection{Elongation de la chaine latérale}

Beaucoup de glucosinolates ne sont pas directement dérivés d'acides aminés, mais à partir des dérivés correspondants. Ceux-ci sont synthétisés par un allongement de la chaîne latérale de l'acide aminé. Chez A. thaliana, la biosynthèse des glucosinolates aliphatiques est initiée par plusieurs cycles d'élongation de la méthionine (Figure 3 (1)). Tout d'abord, la méthionine subit une désamination par l'aminotransférase 4 (BCAT4) dans le cytosol. Le produit résultant de cette désamination, l'acide 2-oxo est ensuite transporté dans le plastide par BAT5 (Gigolashvili et al., 2009). Dans le plastide l'acide 2-oxo est condensé avec l'acétyl-CoA par une méthylthioalkylmalate synthase (MAM1, MAM2, MAM3), suivis d'une réaction d'isomérisation catalysée par une isopropylamalate isomérase 
(IPMI) et d'une décarboxylation oxydative par une isopropylmalate déhydrogénase (IPMDH). Après ces réactions, la molécule peut être transaminée par l'aminotransférase 3 (BCAT3) pour produire l'homométhionine qui peut entrer dans la voie de biosynthèse de la structure glucosinolate ou continuer dans un autre cycle d'élongation de la chaine où les groupes méthylènes sont ajoutés à la chaine latérale au fur et à mesure pour produire des molécules à longue chaine dérivés de la méthionine. (Fahey et al., 2001 ; Halkier et Gershenzon, 2006 ; Grubb et Abel, 2006).

\subsection{Biosynthèse de la structure de base de glucosinolate}

La biosynthèse de la structure des glucosinolates est commune à tous les glucosinolates (Halkier et Gershenzon, 2006), et pendant ce temps la plupart des intermédiaires de cette voie sont connues (Wittstock et Halkier, 2002 ; Halkier et Gershenzon, 2006 ; Sonderby et al., 2010). Cette étape de biosynthèse des glucosinolates prend place dans le cytosol et est catalysée par les enzymes à cytochrome P450 de la famille des CYP79s. En effet, le CYP79A2 catalyse la conversion de la phénylalanine, le CYP79B2 et CYP79B3 catalysent les dérivés de tryptophane et le CYP79F1 et CYP79F2 catalysent les dérivés de la méthionine (Halkier et Du, 1997 ; Mikkelsen et al., 2000 ; Hansen et al., 2001a ; Tantikanjana et al., 2001; Wittstock et Halkier 2002 ; Mikkelsen et al., 2003 ; Naur et al., 2003a ; Halkier et Gershenzon, 2006). Ensuite, les aldoximes sont transformés par oxydation en nitriles ou en composés aci-nitro par les enzymes a cytochrome P450 de la famille des CYP83s, CYP83A1 et CYP83B1 qui ont été identifiés chez $A$. thaliana (Bak et al., 1999 ; Hansen et al., 2001b ; Bak et al., 2001 ; Naur et al., 2003b). Une réaction de conjugaison a eu lieu entre les composés actifs et un donneur du soufre (Cystéine ou glutathion) pour produire le $S$-alkylthiohydroximates. Ce dernier est ensuite converti à son tour en thiohydroximates par clivage de la liaison C-S par une enzyme $C$-S lyase (SUR1) (Mikkelsen et al., 2004). Les glucosyltransférases de la famille des UGT74 et les thiohydroximates donnent lieu aux desulfoglucosinolates. Ces derniers sont ensuite sulfatés dans la dernière étape par les sulfotransférases pour former les glucosinolates (Grubb et al., 2004 ; Grubb et Abel, 2006 ).

\subsection{Modification de la chaine latérale}

Après la formation de la structure de base des glucosinolates, une large gamme de modifications peut se produire au niveau de la chaîne latérale ainsi que dans la fraction glucose qui contribue à une grande variété des glucosinolates (Figure 3). Ces modifications comprennent l'oxydation, l'hydroxylation, la méthoxylation, la désaturation, la sulfatation et la glycosylation. En particulier, les chaînes aliphatiques dérivant de la méthionine sont fortement modifiées. La plupart des variations structurales 
de ces chaînes aliphatiques n'est pourtant due qu'à la variation génétique de trois loci : $G S-O X, G S$-AOP et $G S-O H$ (Mithen et al., 1995 ; Kliebenstein et al., 2001c). Les allèles du locus $G S-O X$ sont responsables de l'oxydation du groupe méthylthioalkyle en méthylsulphinylalkyle. Les allèles $G S-A L K$ et $G S-O H P$ du locus GS-AOP sont responsables respectivement de la désaturation du méthylsulphinylalkyle et le remplacement du groupe méthylsulphinylalkyle par un groupe hydroxy. Finalement, le locus $G S-O H$ assure l'insertion d'un groupe hydroxy supplémentaire pour les alkényle glucosinolates (Kliebenstein et al., 2001a). La plupart des allèles de ces trois loci sont spécifiques de la longueur de la chaine latérale, ce qui explique comment ces loci peuvent engendrer une importante diversité des glucosinolates. La flavine monooxygénase $\mathrm{FM}_{\mathrm{GS}-\mathrm{Ox} 1 \text {, localisé dans le locus }}$ GS-OX a été identifié comme un candidat pour la $S$-oxygénation basé sur la coexpression des gènes de glucosinolates aliphatiques (Hansen et al., 2007). Chez Arabidopsis, sept flavines monooxygénases ont été identifiées et catalysent la réaction de $S$-oxygenation de méthylthioalkyle glucosinolates en méthylsulfinylalkyle glucosinolates. En effet, les monooxygénases FM $\mathrm{GS}$ ox1 à FM $\mathrm{FMS}_{\mathrm{GS} 4}$ et $\mathrm{FM}_{\mathrm{GS}-\mathrm{OX} 6}$ et $\mathrm{FM}_{\mathrm{GS}-\mathrm{OX} 7}$ sont capables de catalyser l'oxygénation de la courte et longue chaine des glucosinolates aliphatiques, néanmoins, $\mathrm{FM}_{\mathrm{GS}-\mathrm{OX} 5}$ est spécifique pour les réactions à longues chaines (Hansen et al., 2007 ; Kong et al., 2016).

Concernant la modification des glucosinolates indoliques, chez $A$. thaliana, les gènes nommé $C Y P 81 F 1, C Y P 81 F 2$ et $C Y P 81 F 3$ de la famille CYP81 ont été identifiés comme gènes responsables de la réaction de l'hydroxylation de 1'indolyl-3-méthyle (I3M) en 4-hydroxy-indolyl-3méthyle (4OH-I3M), le gène CYP81F4 est impliqué dans la de conversion de I3M en 1-hydroxy-indolyl-3-méthyle (1OH-I3M) (Pfalz et al., 2009 ; Pfalz et al., 2011; Pfalz et al., 2016). Une autre famille de gènes, codant pour des indoles glucosinolates $O$-méthyltransférases (IGMT) joue également un rôle dans la modification de la chaine latérale des glucosinolates indoliques. En effet, il a été montré que chez Arabidopsis, les enzymes IGMT1 et IGMT2 peuvent catalyser la conversion de 4OH-I3M en 4-methoxy-indolyl-3-méthyle (4MO-I3M) (Pfalz et al., 2011). Récemment, la fonction du gène IGMT5 a été dévoilée ; le enzyme est responsable de la conversion de 1-hydroxy-indolyl-3-méthyle en 1-methoxy-indolyl-3méthyle (Pfalz et al., 2016).

\section{Variation naturelle de la structure des glucosinolates}

Les rôles divers que les glucosinolates peuvent jouer sont contrôlés par des variations dans leurs structures finales et par leur quantité, cette variation est contrôlée par de nombreux loci quantitatifs et qualitatifs (Magrath et al., 1994 ; Parkin et al., 1994b ; Mithen et al., 1995 ; 
Kliebenstein et al., 2001b ; Kroymann et al., 2003). Cette variation existe chez un grand nombre d'espèces, dont Brassicas est contrôlée le plus souvent par des gènes homologues dans chaque espèce (Magrath et al., 1994 ; Parkin et al., 1994a ; Mithen et al., 1995 ; Heidel et al., 2006 ; Windsor et al., 2005). Les quatres loci contrôlant la variation structurale chez Brassica au niveau des glucosinolates dérivés de la méthionine, GSL-ELONG, -AOP, -OH et $O X$, (Figure 4) fonctionnent épistatiquement pour générer un système génétique modulable pour faire de la sorte que les quatre loci permettent à la plante de générer 14 profils structuraux différents (Kliebenstein et al., 2001c). Les allèles de ces quatre loci structurels expliquent une variation significative des glucosinolates aliphatiques chez la plupart des plantes crucifères telles que Arabidopsis sp (Rodman et al., 1980 ; Magrath et al., 1994 ; Windsor et al., 2005). Des études QTL (locus de caratères quantitatifs) ont également identifié un nombre de loci en corrélation avec l'accumulation des glucosinolates indoliques (Kliebenstein et al., 2001c ; Pfalz et al., 2009). Un locus IGMT1 a été identifié en relation avec les glucosionlates indoliques modifiés au niveau du carbone quatre chez $A$. thaliana (Pfalz et al., 2009). L'enzyme codée par CYP81F2, une monooxygénase du cytochrome P450, catalyse la conversion des $1 \mathrm{OH}-\mathrm{I} 3 \mathrm{M}$ en 1MO-I3M (Pfalz et al., 2009). Un locus indole glucosinolate modifier 1 $(I G M 1)$ a été identifié en relation avec les glucosinolates indoliques modifiés chez A. thaliana, en plus d'un gène sous jacent, $C Y P 81 F 2$ qui a été cloné (Pfalz et al., 2009). Le locus IGMI contribue à la défense contre les pucerons généralistes, ainsi qu'au développement des méchanismes de défense contre les pathogènes non hôtes chez A. thaliana (Pfalz et al., 2009 ; Bednarek et al., 2009 ; Clay et al., 2009). 
Méthionine

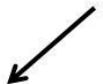

Méthylthiopropyle

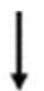

Méthylsulphinylpropyle

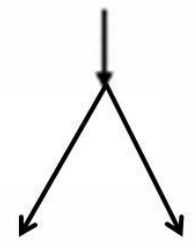

Propényle

Hydrxybutényle

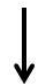

Méthylthiobutyle

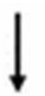

Méthylsulphinylbutyle

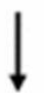

Butényle

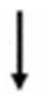

Hydrxybutényle

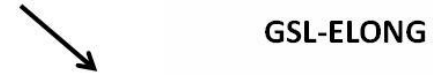

Méthylthiopentyle<smiles>[Y]O[Si][Ca]</smiles>

Méthylsulphinylpentyle

GSL-AOP

Pentényle

GSL-OH

Figure 4: Modèle simplifié de controle de la voie des glucosinolates dérivés de la méthionine chez Brassica

\section{Régulation des glucosinolates}

L'analyse des profils des glucosinolates, leur biosynthèse, leur distribution et leur dégradation dans les organes de plantes a révélé un réseau complexe sur le contrôle de la régulation de ces processus en réponse à des stimuli environnementaux (Petersen et al., 2002). Les études menées sur Arabidopsis en ce qui concerne le gain et la perte de la fonction ont montrés que la biosynthèse des glucosinolates est régulée à différents niveaux et induite par plusieurs voies de signalisation de défense chez la plante (Mikkelsen et al., 2002 ; Halkier et Gershenzon, 2006). Chez A. thaliana, les variations naturelles de la composition en glucosinolates, leur distribution et leur dégradation sont génétiquement contrôlées par plusieurs loci a traits quantitatifs (QTL). En outre, la production des glucosinolates induite par le stress est liée à un réseau de voie de signalisation, telles que les voies de Méthyle Jasmonate (MeJa), de l'Acide Salicylique (SA) et de l'Ethylène (ET) (Brader et al., 2001 ; Kliebenstein et al., 2002). L'analyse des mutants des voies de signalisation hormonale de MeJa, SA et de ET et leurs réponses au stress biotique a révélé des interactions complexes entre les différentes voies de signalisation hormonale et la régulation de la biosynthèse des glucosinolates (Mikkelsen et al., 2003), étant donné que le traitement exogène avec MeJa avait conduit à l'accumulation des glucosinolates aliphatiques et indoliques (Brader et al., 2001), et le traitement avec SA conduit à une accumulation de certains glucosinolates indoliques et à la 
répression de la biosynthèse des glucosinolates aliphatiques (Kliebenstein $e t$ al., 2002).

Plusieurs protéines nucléaires ont été récemment identifiées comme des régulateurs de métabolisme de glucosinolates (Figure 5). Les facteurs de transcription MYB28, MYB29 et MYB76 ont été identifiés, comme régulateurs positifs de la biosynthèse des glucosinolates aliphatiques (Hirai et al., 2007 ; Sønderby et al., 2007, 2010). Les gènes MYB29 et MYB76 réglementent la quantité de glucosinolates aliphatiques à courte chaine (Sønderby et al., 2007 ; Gigolashvili et al., 2007, 2008) tandis que MYB28 joue un rôle majeur dans le contrôle de la quantité de glucosinolates aliphatiques à longue et à courte chaine (Hirai et al., 2007 ; Sønderby et al., 2007 ; Gigolashvili et al., 2009). La double mutation de myb28 / myb29 est presque dépourvue de glucosinolates aliphatiques, vraisemblablement par des effets épistatiques (Sønderby et al., 2007). Par contre, il n'y avait pas de changement dans le niveau des glucosinolates indoliques quand MYB28, MYB29 et/ou MYB76 sont inactivées (Sønderby et al., 2007), ce qui montre donc le rôle exclusif de MYB28, MYB29 et MYB76 dans la voie de biosynthèse des glucosinolates aliphatiques. D'un autre côté, il a été montré que les facteurs de transcription, MYB34, MYB51 et MYB122 ont une régulation positive sur la transcription des gènes de biosynthèse de tryptophane et de glucosinolates idoliques et l'auxine (IAA). Il a été montré recemment qu'une autre famille de facteurs de transcription appelée WRKY ont un rôle dans la régulation des glucosinolates indoliques. En effet, la double mutation des gènes wrkyl8 / wrky40 réduit l'expression des gènes MYB34 et CYP79B2 (Chi et al., 2013) 


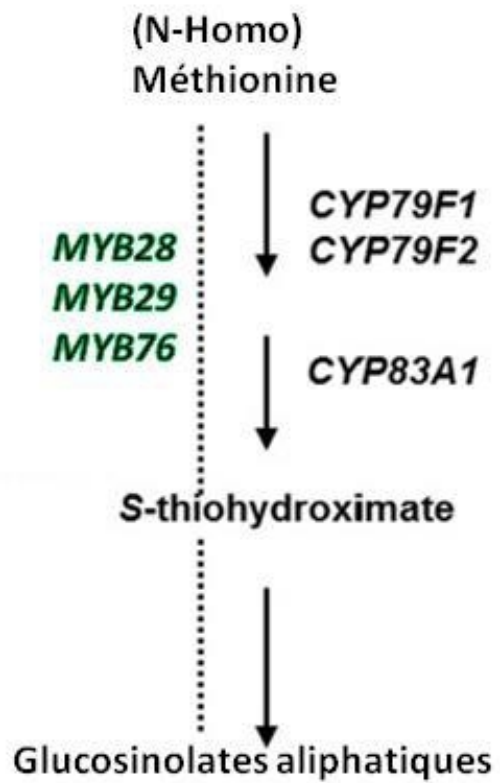

Tryptophane
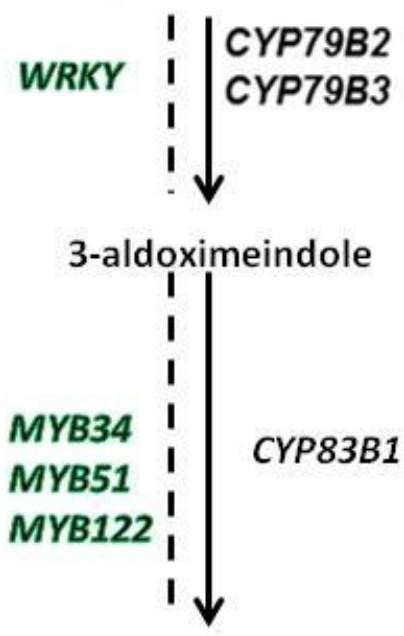

Glucosinolates indoliques

Figure 5. Régulation de la voie de biosynthèse des glucosinolates par des facteurs de transcriptions.

La figure montre les facteurs de transcription, MYB28, MYB29 et MYB76 qui régulent la voie de biosynthèse des glucosionolates aliphatiques, en agissant sur les gènes CYP79F1, CYP79F2 et CYP83A1 et les facteurs de transcription, WRKY, MYB34, MYB51et MYB122 et qui régulent la voie de biosynthèse des glucosinolates indoliques en agissant sur les gènes CYP79B2, CYP79B3 et CYP83B1 (Chi et al., 2013; Gigolashvili et al., 2007 ; Sønderby et al., 2007).

\section{Conclusion}

Les glucosinolates sont des derivés biologiquement actifs, ils interviennent dans les réactions de défense des plantes contre le stress biotique et abiotique. L'indisponibilité des ressources génomiques et l'existence d'une redondance des gènes due à la nature polyploïde de la majorité d'espèces Brassica ont largement limité les connaissances sur les gènes de biosynthèse des glucosinolates et leur régulation. Au cours de ces dernières années, des progrès significatifs ont été notés dans la compréhension des processus de biosynthèse et de régulation des glucosinolates chez les plantes à travers l'accélération des projets de séquençage à haut débit, de plus l'étude des processus de biosynthèse des glucosinolates s'est étendue vers d'autre espèces non modèles et même dans des familles autres que les Brassicacae, telles que les Cleomaceae, les Capparaceae, les Resedaceae... de l'ordre des Brassicales. L'activation des glucosinolates suite à une attaque externe et les propriétés de leurs produits 
d'hydrolyses (isothiocyanates, thiocyanates et nitriles) ont suggérés depuis longtemps que la fonction principale de ces composés chez les plantes est de se défendre contre les herbivores et les agents pathogènes. Le système glucosinolate-myrosinase est la clé de la relation plante-attaque externe. Pour cela, il a fait objectif de plusieurs études pour comprendre les mécanismes d'action des produits d'hydrolyses et comment ils exercent leur toxicité sur les plantes. Plusieurs études ont signalé que les plantes réagissent aux dommages causés par les insectes en accumulant systématiquement des concentrations plus élevées de glucosinolates, cela a permet 1'augmentation de la résistance des plantes aux attaques ultérieures. De plus, les plantes ont acquis des capacités dans la modulation de leurs niveaux en glucosinolates pour gérer au mieux les attaques des insectes et des agents pathogènes, ceci a été montré par une ou plusieurs enzymes de biosynthèse, comme les CYP79 qui catalysent la conversion d'acides aminés en aldoximes tout en fournissant des outils moléculaire importants pour moduler la teneur en glucosinolates des plantes. En outre, des connaissances sur les facteurs qui régulent les efflux à travers les voies de la biosynthèse et la dégradation ont permis de comprendre comment l'accumulation des glucosinolates en fonction de l'attaque est contrôlée à plusieurs niveaux en réponse à des stimuli environnementaux, ceci est assuré par des protéines régulatrices, telle que les facteurs de transciption MYB qui régulent spécifiquement la quantité des glucosinolates. La diversification des glucosinolates a été étudiée par la variation naturelle de la structure des glucosinolates qui est controlée par plusieurs loci quantitatifs et qualitatifs. Cette révolution a été fortement soutenue par le développement des outils génomiques et la mise au point des technologies biologiques qui relient les données génétiques, protéiques et métaboliques en permettant la compréhension des mécanismes biochimiques et moléculaires de biosynthèse, de dégradation et de régulation des glucosinolates mais aussi leur variation naturelle en permettant aux plantes de faire face aux attaques des ennemis naturels.

\section{References :}

1. Agerbirk N, Olsen CE. (2012). Glucosinolate structures in evolution. Phytochemistry. 77 : 16-45.

2. Bak S, Olsen CE, Petersen BL, Møller BL, Halkier BA. (1999). Metabolic engineering of p-hydroxybenzylglucosinolate in Arabidopsis by expression of the cyanogenic CYP79A1 from Sorghum bicolor. Plant J. 20 : 663-671.

3. Bak S, Tax FE, Feldmann KA, Galbraith DW, Feyereisen R. (2001). CYP83B1, a cytochrome P450 at the metabolic branch point in auxin and indole glucosinolate biosynthesis in Arabidopsis. Plant Cell. 13: 101-111. 
4. Bednarek P, Pislewska-Bednarek M, Svatoš A, Schneider B, Doubskỳ J, Mansurova M, Humphry M, Consonni C, Panstruga R, SanchezVallet A, Molina A, Schulze-Lefert P. (2009). A glucosinolate metabolism pathway in living plant cells mediates broad-spectrum antifungal defense. Science. $323: 101-106$.

5. Bennett R, Ludwig-Müller J, Kiddle G, Hilgenberg W, Wallsgrove R. (1995). Developmental regulation of aldoxime formation in seedlings and mature plants of Chinese cabbage (Brassica campestris) and oilseed rape (Brassica napus): glucosinolate and IAA biosynthetic enzymes. Planta. 196 : 239-244.

6. Block G, Patterson B, Subar A. (1992). Fruit, vegetables, and cancer prevention: A review of the epidemiological evidence. Nutr. Cancer. $18: 1-29$

7. Bones, Slupphaug G. (1989). Purification, characterization and partial amino acid sequencing of J-thioglucosidase from Brassica napus. Plant Physiol. 134 : 722 - 729

8. Brader G, Mikkelsen MD, Halkier BA, Tapio Palva E. (2006). Altering glucosinolate profiles modulates disease resistance in plants. The Plant Journal. 46 : 758-767.

9. Brader G, Tas E, Palva ET. (2001). Jasmonate-dependent induction of indole glucosinolates in Arabidopsis by culture filtrates of the nonspecific pathogen Erwinia carotovora. Plant Physiol. 126 : 849860

10. Brown PD, Tokuhisa JG, Reichelt M, Gershenzon J. (2003). Variation of glucosinolate accumulation among different organs and developmental stages of Arabidopsis thaliana. Phytochemistry. 62 : 471-481

11. Chen S, and Andreasson E. (2001). Update on glucosinolate metabolism and transport. Plant Physiol. Biochem. 39 : 743-758.

12. Chi Y, Yang Y, Zhou Y, Zhou J, Fan B, Yu JQ, Chen Z. (2013). Protein-protein interactions in the regulation of WRKY transcription factors. Mol Plant. 6 : 287-300

13. Clay NK, Adio AM, Denoux C, Jander G, Ausubel FM. (2009). Glucosinolate metabolites required for an Arabidopsis innate immune response. Science. 323 : 95-101.

14. Croteau R, Kutchan TM, Lewis NG. (2000). Natural products (secondary metabolites). Biochemistry and molecular biology of plants. 24 : 1250-1319.

15. Daxenbichler ME, Spencer GF, Carlson DG, Rose GB, Brinker AM, Powell RG. (1991). Glucosinolate Composition of Seeds from 297 Species of Wild Plants. Phytochemistry. 30 : 2623-2638 . 
16. De Vos M, Kriksunov KL, Jander G. (2008). Indole-3-acetonitrile production from indole glucosinolates deters oviposition by Pieris rapae. Plant Physiol. 146 : 916-926.

17. Fahey JW, Zalcmann AT, Talalay P. (2001). The chemical diversity and distribution of glucosinolates and isothiocyanates among plants. Phytochemistry. $56: 5-51$.

18. Fenwick GR, Heaney RK, Mullin, WJ. (1983). Glucosinolates and their breakdown products in foods and food plants. CRC Crit. Ret'. Fd Sci. Nutr. 18 : 123-201.

19. Gigolashvili T, Engqvist M, Yatusevich R, Müller C, Flügge UI. (2008). HAG2/MYB76 and HAG3/MYB29 exert a specific and coordinated control on the regulation of aliphatic glucosinolate biosynthesis in Arabidopsis thaliana. New Phytologist. 177 : 627-642

20. Gigolashvili T, Yatusevich R, Berger B, Müller C, Flügge UI. (2007). The R2R3-MYB transcription factor HAG1/MYB28 is a regulator of methionine-derived glucosinolate biosynthesis in Arabidopsis thaliana. The Plant Journal. $51: 247-261$

21. Gigolashvili T, Yatusevich R, Rollwitz I, Humphry M, Gershenzon J, Fluegge UI. (2009). The plastidic bile acid transporter 5 is required for the biosynthesis of methionine-derived glucosinolates in Arabidopsis thaliana. Plant Cell. 21: 1813-1829.

22. Grubb CD, Abel S, (2006). Glucosinolate metabolism and its control. Trends Plant Sci. 11: 89-100.

23. Grubb CD, Zipp BJ, Ludwig-Mu“ller J, Masuno MN, Molinski TF, Abel S. (2004). Arabidopsis glucosyltransferase UGT74B1 functions in glucosinolate biosynthesis and auxin homeostasis. The Plant Journal. 40 : 893-908.

24. Halkier BA, Du L. (1997). The biosynthesis of glucosinolates. Trends Plant Sci. 11: 425-430.

25. Halkier BA, Gershenzon J. (2006). Biology and biochemistry of glucosinolates. Annu. Rev. Plant Biol. 57 : 303-333.

26. Hansen BG, Kliebenstein DJ, Halkier BA. (2007). Identification of a flavin-monooxygenase as the S-oxygenating enzyme in aliphatic glucosinolate biosynthesis in Arabidopsis. Plant J. 50 : 902-910

27. Hansen CH, Du L, Naur P, Olsen CE, Axelsen KB, Hick A.J. Pickett JA, Halkier BA. (2001b). CYP83B1 is the oxime-metabolizing enzyme in the glucosinolate pathway in Arabidopsis. J Biol Chem. 276 : 24790-24796

28. Hansen CH, Wittstock U, Olsen CE, Hick AJ, Pickett JA, Halkier BA. (2001a). Cytochrome P450 CYP79F1 from Arabidopsis catalyzes the conversion of dihomomethionine and trihomomethionine to the 
corresponding aldoximes in the biosynthesis of aliphatic glucosinolates. J. Biol. Chem. 276 : 11078-11085.

29. Hartmann T. (2007). From waste products to ecochemicals: Fifty years research of plant secondary metabolism. Phytochemistry. 68 : 28312846.

30. Hasegawa T, Yamada K, Kosemura S, Yamamura S, Hasegawa K. (2000). Phototropic stimulation induces the conversion of glucosinolate to phototropism-regulating substances of radish hypocotyls. Phytochemistry. 54 : 275-279.

31. Heidel AJ, Clauss MJ, Kroymann J, Savolainen O, and MitchellOldsTh. (2006). Natural Variation in MAM Within and Between Populations of Arabidopsis lyrata Determines Glucosinolate Phenotype. Genetics. 173 : 1629-1636

32. Hirai MY, Klein K, Fujikawa Y, Yano M, Goodenowe DB, Yamazaki Y, Kanaya S, Nakamura Y, Kitayama M, Suzuki H. (2005). Elucidation of gene-to-gene and metabolite-to-gene networks in Arabidopsis by integration of metabolomics and transcriptomics. $J$ Biol Chem. 280 : 25590-25595.

33. Hirai, MY, Sugiyama K, Sawada Y, Tohge T, Obayashi T, Suzuki A. (2007). Omics-based identification of Arabidopsis Myb transcription factors regulating aliphatic glucosinolate biosynthesis. Proc. Natl Acad. Sci. USA. 104 : 6478-6483.

34. Howe GA, Jander G. (2008). Plant immunity to insect herbivores. Annu Rev Plant Biol. 59 : 41-66.

35. Iori R, Leon O, Fontana F, Lazzeri L, Palmeri S, Attene G, Ciriciofolo E, Leto C, Mosca G. (1990). Evoluzione dei principali costituenti qualitativi durante il riempimento e la maturazione del seme di colza (Brassica napus L.) in alcuni ambienti di coltivazione. Riv. Agronomia. 24 : 243-249.

36. Iriti M, Faoro F. (2009). Chemical diversity and defence metabolism: how plants cope with pathogens and ozone pollution. International Journal of Molecular Sciences. 10 : 3371-3399.

37. Iversen TH, Baggerud C. (1980). Myrosinase activity in differentiated and undifferentiated plants of Brassicaceae Zeitschr. Pflanzenphys. 97 : 399-407.

38. Kimura M, Anzai H, Yamaguchi I. (2001). Microbial toxins in plantpathogen interactions: Biosynthesis, resistance mechanisms, and significance. Journal of General and Applied Microbiology. 47: 149160.

39. Kliebenstein D, Pedersen D, Barker B, Mitchell-Olds T, (2002). Comparative analysis of quantitative trait loci controlling 
glucosinolates, myrosinase and insect resistance in Arabidapsis thaliana. Genetics. $1618: 325-332$.

40. Kliebenstein DJ, Gershenzon J, Mitchell-Olds T, (2001b). A Comparative quantitative trait loci mapping of aliphatic, indolic and benzylic glucosinolate production in Arabidopsis thaliana leaves and seeds. Genetics. 159 : 359-370.

41. Kliebenstein DJ, Kroymann J, Mitchell-Olds T. (2005). The glucosinolate myrosinase system in an ecological and evolutionary context. Curr. Opin. Plant Biol. 8 : 264-271.

42. Kliebenstein DJ, Lambrix VM, Reichelt M, Gershenzon J, and Mitchell-Olds T. (2001a). Gene duplication and the diversification of secondary metabolism: side chain modification of glucosinolates in Arabidopsis thaliana. Plant Cell 13 : 681-693.

43. Kliebenstein DJ, Kroymann J, Brown P, Figuth A, Pedersen D, Gershenzon J, Mitchell-Olds T. (2001c). Genetic control of natural variation in Arabidopsis glucosinolate accumulation. Plant Physiol. $126: 811-825$.

44. Kong W, Li J, Yu Q, Cang W, Xu R, Wang Y, Ji W. (2016). Two Novel Flavin- Containing Monooxygenases Involved in Biosynthesis of Aliphatic Glucosinolates. Frontiers in plant science. 7 : 1292.

45. Kroymann J, Donnerhacke S, Schnabelrauch D, Mitchell-Olds T. (2003). Evolutionary dynamics of an Arabidopsis insect resistance quantitative trait locus. Proc. Natl. Acad. Sci. USA 100 : 14587-14592.

46. Lambrix VM, Reichelt M, Mitchell-Olds T, Kliebenstein DJ, Gershenzon J. (2001). The Arabidopsis epithiospecifier protein promotes the hydrolysis of glucosinolates to nitriles and influences Trichoplusia ni herbivory. Plant Cell, 13: 2793-2807.

47. London SJ, Yuan JM, Chung FL, Gao YT, Coetzee GA, Ross RK, Yu MC. (2000). Isothiocyanates, glutathione S-transferase M1 and T1 polymorphisms, and lung-cancer risk: a prospective study of men in Shanghai, China. Lancet. 356 : 724-29.

48. Magrath R, Bano F, Morgner M, Parkin I, Sharpe A, Lister C, Dean C, Turner J, Ludiate D, Mithen R. (1994). Genetics of aliphatic glucosinolates: I. Side chain elongation in Brassica napus and Arabidopsis thaliana. Heredity. 72 : 290-299.

49. Mewis I, Ulrich CH, Schnitzler WH. (2002). The role of glucosinolates and their hydrolysis products in oviposition and host-plant finding by cabbage webworm, Hellula undalis. Entomologia experimentalis applicata. 105 : 129-139.

50. Mikkelsen MD, Hansen CH, Wittstock U, Halkier BA. (2000). Cytochrome P450 CYP79B2 from Arabidopsis catalyzes the conversion of tryptophan to indole-3-acetaldoxime, a precursor of 
indole glucosinolates and indole-3-acetic acid. J Biol Chem. 275: 33712-33717.

51. Mikkelsen MD, Naur P, Halkier BA. (2004). Arabidopsis mutants in the $\mathrm{C}-\mathrm{S}$ lyase of glucosinolate biosynthesis establish a critical role for indole-3-acetaldoxime in auxin homeostasis. Plant J. 37 : 770-777.

52. Mikkelsen MD, Petersen BL, Glawischnig E, Jensen AB, Andreasson E, Halkier BA. (2003). Modulation of CYP79 genes and glucosinolate profiles in Arabidopsis by defense signaling pathways. Plant Physiol. 131 : 298-308.

53. Mikkelsen MD, Petersen BL, Olsen CE, Halkier BA. (2002). Biosynthesis and metabolic engineering of glucosinolates. Amino Acids. 22 : 279-95.

54. Miles CI, Del Campo ML, Renwick JA. (2005). Behavioral and chemosensory responses to a host recognition cue by larvae of Pieris rapae. J Comp Physiol Neuroethol Sens Neural Behav Physiol. 191 : 147-155.

55. Mithen R, Clarke J, Lister C, Dean C. (1995). Genetics of aliphatic glucosinolates. III. Side chain structure of aliphatic glucosinolates in Arabidopsis thaliana. Heredity. 74 : 210-215.

56. Mithen R, Faulkner K, Magrath R, Rose P, Williamson G, Marquez J. (2003). Development of isothiocyanate-enriched broccoli, and its enhanced ability to induce phase 2 detoxification enzymes in mammalian cells. Theor. Appl. Genet. 106 : 727-734.

57. Naur P, Hansen CH, Bak S, Hansen BG, Jensen NB, Nielsen HL, Halkier BA, (2003a). CYP79B1 from Sinapis alba converts tryptophan to indole-3-acetaldoxime. Arch Biochem Biophys. 409 : 235-241.

58. Naur P, Petersen BL, Mikkelsen MD, Bak S, Rasmussen H, Olsen CE, Halkier BA. (2003b). CYP83A1 and CYP83B1, two non redundant cytochrome P450 enzymes metabolizing oximes in the biosynthesis of glucosinolates in Arabidopsis. Plant Physiol. 133 : 63-72.

59. Newman, DJ, Cragg GM. (2012). Natural Products as Sources of New Drugs over the 30 Years from 1981 to 2010. Journal of Natural Products. $75: 311-335$.

60. Parkin I, Magrath R, Keith D, Sharpe A, Mithen R, Lydiate D. (1994b). Genetics of aliphatic glucosinolates. II. Hydroxylation of alkenyl glucosinolates in Brassica napus. Heredity. 72: 594-598.

61. Petersen BL, Chen S, Hansen CH, Olsen CE, Halkier BA, (2002). Composition and content of glucosinolates in developing Arabidopsis thaliana. Planta, 214 : 562-571.

62. Pfalz M, Mikkelsen MD, Bednarek P, Olsen CE, Halkier BA, Kroymann J. (2011). Metabolic engineering in Nicotiana benthamiana 
reveals key enzyme functions in Arabidopsis indole glucosinolate modification. Plant Cell. 23 : 716-729.

63. Pfalz M, Mukhaimar M, Perreau F, Kirk J, Hansen CIC, Olsen CE, Agerbirk N, Kroymann J. (2016). Methyl Transfer in Glucosinolate Biosynthesis Mediated by Indole Glucosinolate O-Methyltransferase 5. Plant physiology. 172 : 2190-2203.

64. Pfalz M, Vogel H, Kroymann J. (2009). The gene controlling the Indole Glucosinolate Modifier1 quantitative trait locus alters indole glucosinolate structures and aphid resistance in Arabidopsis. Plant Cell. 21 : 985-999.

65. Ratzka A, Vogel H, Kliebenstein DJ, Mitchell-Olds T, Kroymann J. (2002). Disarming the mustard oil bomb. Proc Natl Acad Sci. 99 : 11223-11228. ,

66. Rausch T, Butcher DN, Hilgenberg W. (1983). Indole3methylglucosinolate biosynthesis and metabolism in clubrootdiseased plants. Physiologia Plantarum. 58 : 93-100.

67. Redovnikovic IR, Glivetic T, Delonga K, Vorkapic-Furac J. (2008). Glucosinolates and their portential role in plant. Period. Biol. 110 : 297-309.

68. Reintanz B, Lehnen M, Reichelt M, Gershenzon J, Kowalczyk M, Sandberg G, Godde M, Uhl R, and Palme K. (2001). Bus, a bushy Arabidopsis $C Y P 79 F 1$ knockout mutant with abolished synthesis of short-chain aliphatic glucosinolates. The Plant Cell. 13 : 351-367.

69. Richter G. (1993). Métabolisme des végétaux. Physiologie et Biochimie. Ed. Presses Polytechniques et Universitaire Romandes. 322-323.

70. Robiquet PJ, Boutron F. (1931). Sur la semence de moutarde. J Pharm Chim. 17 : 279-282.

71. Rodman JE, Kruckeberg AR, Al-Shehbaz IA. (1981). Chemotaxonomic diversity and complexity in seed glucosinolates of Caulanthus and Streptanthus (Cruciferae). Syst. Bot. 6 : 197-222.

72. Rodman JE. (1980). Population variation and hybridization in sea rockets (Cakile, Cruciferae): Seed glucosinolate characters. Am. J. Bot. 67 : 1145-1159.

73. Sang TP, Truscott RJW. (1984). Liquid chromatographic determination of glucosinolates in rapeseed as desulfoglucosinolates $\mathrm{J}$. Assoc. Off. Anal. Chem. 67 : 829-833.

74. Schnug E. (1990). Glucosinolates fundamental, environmental and agricultural aspects, in Sulfur Nutrition and Sulfur Assimilation in Higher Plant; Fundamental, Environmental and Agricultural Aspects. The Hague: SPB Academic Publishing. 97-106.

75. Sønderby IE, Geu-Flores F, Halkier BA. (2010). Biosynthesis of 
glucosinolates gene discovery and beyond. Trends in Plant Science. 15 : 283-290

76. Sønderby IE, Hansen BG, Bjarnholt N, Ticconi C, Halkier BA, Kliebenstein DJ. (2007). A systems biology approach identifies a R2R3 MYB gene subfamily with distinct and overlapping functions in regulation of aliphatic glucosinolates. PLoS One. 2 : e1322.

77. Sun JY, Sønderby IE, Halkier BA, Jander G, de Vos M, (2009). Nonvolatile intact indole glucosinolates are host recognition cues for ovipositing Plutella xylostella. J. Chem. Ecol. 358 : 1427-1436.

78. Tantikanjana T, Yong JW, Letham DS, Griffith M, Hussain M, Ljung K, Sandberg G, Sundaresan V. (2001). Control of axillary bud initiation and shoot architecture in Arabidopsis through the SUPERSHOOT gene. Genes Dev. 15 : 1577-1588.

79. Tierens KJ, Thomma B, Brower M, Schmidt J, Kistner K, Porzel A, Mauch-Mani B, Cammue B, Broekaert W. (2001). Study of the role of antimicrobial glucosinolate-derived isothiocyanates in resistance of Arabidopsis to microbial pathogens. Plant Physiol. 125 : 1688-1699.

80. Van Poppel G, Verhoeven DT, Verhagen H, Goldbohm RA, (1999). Brassica vegetables and cancer prevention. Epidemiology and mechanisms. Adv. Exp. Med. Biol. 472 : 159-168.

81. Verhoeven DTH, Verhagen H, Goldbohm RA, van den Brandt PA, van Poppel G. (1997). A review of mechanisms underlying anticarcinogenicity by brassica vegetables. Chem Biol Interact. 103 : 79-129.

82. Wang J, Xu L, Wang E. (2009). Robustness and coherence of a threeprotein circadian oscillator: landscape and flux perspectives. Biophys J. 97 : 3038-3046.

83. Windsor AJ, Reichelt M, Figuth A, Svatos A, Kroymann J, Kliebenstein DJ, Gershenzon J, Mitchell-Olds T. (2005). Geographic and evolutionary diversification of glucosinolates among near relatives of Arabidopsis thaliana (Brassicaceae). Phytochemistry. 66 : 13211333.

84. Wittstock U, Halkier BA. (2002). Glucosinolate research in the Arabidopsis era. Trends Plant Sci. 7 : 263-270

85. Zabala MD, Grant M, Bones AM, Bennett R, Lim YS, Kissen R, Rossiter JT. (2005). Characterisation of recombinant epithiospecifier protein and its over-expression in Arabidopsis thaliana. Phytochemistry. $668: 859-867$ 